\title{
ATTEMPTS OF THERMAL IMAGING CAMERA USAGE IN ESTIMATIONS OF THE CONVECTIVE HEAT LOSS FROM A VERTICAL PLATE
}

\author{
Hubert Denda ${ }^{1, a}$, Witold M. Lewandowski ${ }^{1}$, Michał Ryms ${ }^{1}$, Patrycja Wcisło ${ }^{1}$ and Ewa Klugmann-Radziemska ${ }^{1}$ \\ ${ }^{1}$ Gdansk University of Technology, 80-233Gdansk, Poland
}

\begin{abstract}
In this paper a new method for determining heat transfer coefficients using a gradient method has been developed. To verify accuracy of the proposed method vertical isothermal heating plate with natural convection mechanism has been examined. This configuration was deliberately chosen, because of the fact that such case is historically the earliest and most thoroughly studied and its rich scientific documentation - the most reliable. New method is based on temperature field visualization made in perpendicular plane to the heating surface of the plate using infrared camera. Because the camera does not record temperature of air itself but the surface only, therefore plastic mesh with low thermal conductivity has been used as a detector. Temperature of each mesh cell, placed perpendicular to the vertical heating surface and rinsed with convection stream of heated air could be already recorded by infrared camera. In the same time using IR camera surface of heating plate has been measured. By numerical processing of the results matrix temperature gradient on the surface $\partial \mathrm{T} / \partial \mathrm{x} \mid \mathrm{x}=0$, local heat transfer coefficients $\alpha_{\mathrm{y}}$, and local values of Nusselt number $\mathrm{Nu}_{\mathrm{y}}$, can be calculated. After integration the average Nusselt number for entire plate can be calculated. Obtained relation characteristic numbers $\mathrm{Nu}=0.647 \mathrm{Ra}^{0.236}\left(\mathrm{R}^{2}=0.943\right)$, has a good correlation with literature reports and proves usefulness of the method.
\end{abstract}

\section{Nomenclature}

$\mathrm{y}$, coordinate measured in the direction of motion;

$\mathrm{x}$, coordinate measured normal to the direction of motion;

g, acceleration due to gravity;

1, characteristic linear dimension of the phenomenon;

$\mathrm{T}$, temperature;

$\alpha$, heat-transfer coefficient;

$\lambda$, thermal conductivity;

q, heat flux;

$\Delta \mathrm{T}$, temperature difference between the wall and the ambient fluid, $\left(\mathrm{T}_{\mathrm{w}^{-}} \mathrm{T}_{\infty}\right)$;

$\beta$, coefficient of thermal expansion;

a, coefficient of thermal diffusivity;

$v$, kinematic viscosity;

Q, rate of heat exchanged during convection;

A, heat exchange surface;

C, $n$, coefficients for different conditions of heat flow;

\section{Subscripts}

cr, properties corresponding to critical point;

$\infty$, properties evaluated in bulk fluid;

$\mathrm{w}$, properties evaluated at the wall;

g, properties related to main heater

$\mathrm{p}$, properties related to auxiliary heater

\section{Introduction}

Theoretical studies of natural convection, when the fluid considered is air, came down to analytical, asymptotic and numerical solution of Navier-Stokes and Fourier-Kirchhoff equations, together with continuity equations and equation of state for gases. Examples of such researches are presented in the works of: Churchill and Chu [1], Martynenko et al. [2], Hellums et al. [3] Vynnycky et al. [4] and others. Theoretical studies have only been indicated because this work is devoted to experimental issues

Non-invasive techniques such as interferometry [5] can be also used to study convective temperature fields near heated surface, however this technique is more qualitative than quantitative.

We think that experimental methods to determination temperature fields, in which volumetric sensors are used, have several disadvantages and most important is disruption in natural convection flux.

For this reason we decided to develop less invasive, rapid method for simultaneous measurements of entire temperature field using thermal imaging camera. This method allows to remote reading and recording numerical results in form of a matrix (temperature, coordinates).

\footnotetext{
$\overline{{ }^{a} \text { Corresponding author: dendi8@wp.pl }}$
} 
So far infrared camera was used only for surface uniformity research (e.g. Zhao-Zhuang [6]) and to determine local overheating, which indicates place with increased heat loss from buildings, insulation defects, thermal bridges, etc.

\section{Theoretical basis}

Natural convection is a heat transfer mechanism associated with movement of matter in fluids. It consist in temperature and velocity fields interactions. The temperature changes cause differences in density of the fluid, which in effect begins moving under the influence of buoyancy forces. On the other hand, fluid movement intensifies further energy exchange. This mutual feedback continues until it reaches the specific, stabilized temperature, in which the thermodynamic equilibrium of temperature and velocity fields is determined. The Rayleigh number $\boldsymbol{R} \boldsymbol{a}$ determines the intensity of convective heat losses. Critical Rayleigh number $\boldsymbol{R} \boldsymbol{a}_{\boldsymbol{c}}$ specifies a boundary between end of heat transfer caused by conduction, and beginning of more intensive heat transfer caused by convection.

Dimensionless, characteristic Rayleigh number which is a criterion of similarity in natural convection phenomena is defined as:

$$
R a=\frac{g \cdot l^{3} \cdot \beta \cdot \Delta T}{v \cdot a}
$$

The rate of convection heat transfer is conveniently expressed by Newton's law of cooling as:

$$
Q=\alpha \cdot A \cdot \Delta T
$$

Combination of $\lambda$ and $\alpha$ defines second characteristic number in heat exchange phenomena similarities - the Nusselt number:

$$
N u=\frac{\alpha \cdot l}{\lambda}
$$

For natural convection operate Nusselt and Rayleigh's relation is defining as:

$$
N u=C \cdot R a^{n}
$$

Using above equation heat transfer can be described in the following cases: from any configuration of heating surface (plate, sphere, cylinder, cone, etc.), alternating their position in space (Horizontal, Vertical, Inclined), varying intensity of the phenomena (laminar, transitional and turbulent range). Equation (4) allows also comparing and verifying the correctness of theoretical analysis with the experimental results.

To prepare experimental results of natural convection in the form of equation (4) and determine the convective heat losses from real surfaces (walls of buildings, apparatuses, boilers, etc.) of indicated relations, it is necessary to get know the value of convective heat flux (2). There are three methods for determining heat transfer coefficient: gradient, differential and balance one. In following study the gradient method has been used.

Gradient method consists in directly calculations of the temperature field of fluid in vicinity of heated plate. Analysis of this field allows to define temperature gradients distribution around the plate $\partial \boldsymbol{T} /\left.\partial \boldsymbol{x}\right|_{\mathrm{x}=0}$ which is bound to Fourier's equation (5) to a local heat transfer coefficients field $\boldsymbol{\alpha}_{\boldsymbol{y}}$ and Nusselt number $\boldsymbol{N} \boldsymbol{u}_{\boldsymbol{y}}$ (3). Averaging these numbers allows using directly equation (2) to determine convective heat flux.

Assuming that system is in equilibrium steady state and heat transfer from isothermal vertical flat surface is unidirectional, comparing heat fluxes described by Newton's and Fourier's relation gives:

$$
\left.q_{y}=\alpha_{y} \Delta T=-\lambda \cdot \frac{\partial T}{\partial x}\right]_{x=0}
$$

Having regard to equation (3) we finally obtain the relation which from we can directly calculate the Nusselt number:

$$
N u=\frac{\left.-\lambda \cdot \frac{\partial T}{\partial x}\right]_{x=0} \cdot l}{\Delta T}
$$

Error of the method depends on accuracy of temperature distribution in direction normal to heating surface, which is affected by: temperature measurement accuracy, distance and size of the measuring probes.

Additional defects of traditional gradient method are large time-consumption and effort, as well invasiveness because measurement probes placed into convective heat flux disturb its motion and inhibit heat transfer. More observations of discussed methods can be found in the work [7].

\section{The test stand}

\subsection{Heating plate}

Research was conducted on isothermal, vertical heating plate with a width of $247 \mathrm{~mm}$ and height of $495 \mathrm{~mm}$ (aspect ratio 1:2). The picture and partial crosssection of plate are shown in Figure 1.

Presented method of heaters arrangement provides reverse heat rate $\boldsymbol{Q}_{g}$ compensation (one-dimensional heat rate). The purpose of auxiliary heater (150Watts) is reheating main heater ( $300 \mathrm{Watts}$ ), so that the difference in barrier between heaters was $0 \mathrm{~K}$, and $\boldsymbol{Q}_{g}=\boldsymbol{Q}_{\boldsymbol{p}}$. Reverse rate from main heating element is then inhibited and the entire of its power, as the heat flux $\boldsymbol{Q}$, is transmitted to fluid through the convection. Additionally, outer surface of the plate was polished to reduce heat loss caused by radiation.

Varying depth holes, designed for temperature sensors, have been positioned in the three boards' layers. External microprocessor DALLAS DS18B20 sensors have been connected to two 8-channel thermometric AVT5330 measure modules, providing data reading with resolution of $0.1 \mathrm{~K}$ and an accuracy of $0.25 \mathrm{~K}$ in temperature range of $0-80^{\circ} \mathrm{C}$. Sensors were placed on four different altitudes of plate.

Regulation system automatically maintain preset temperature of heating surface by controlling power of main heater and simultaneously power of auxiliary heater so that $\boldsymbol{\Delta} \boldsymbol{T}=0$. After stabilizing temperature of plate and undisturbed area and after stabilizing power of heaters, indications of all sensors were recorded. 


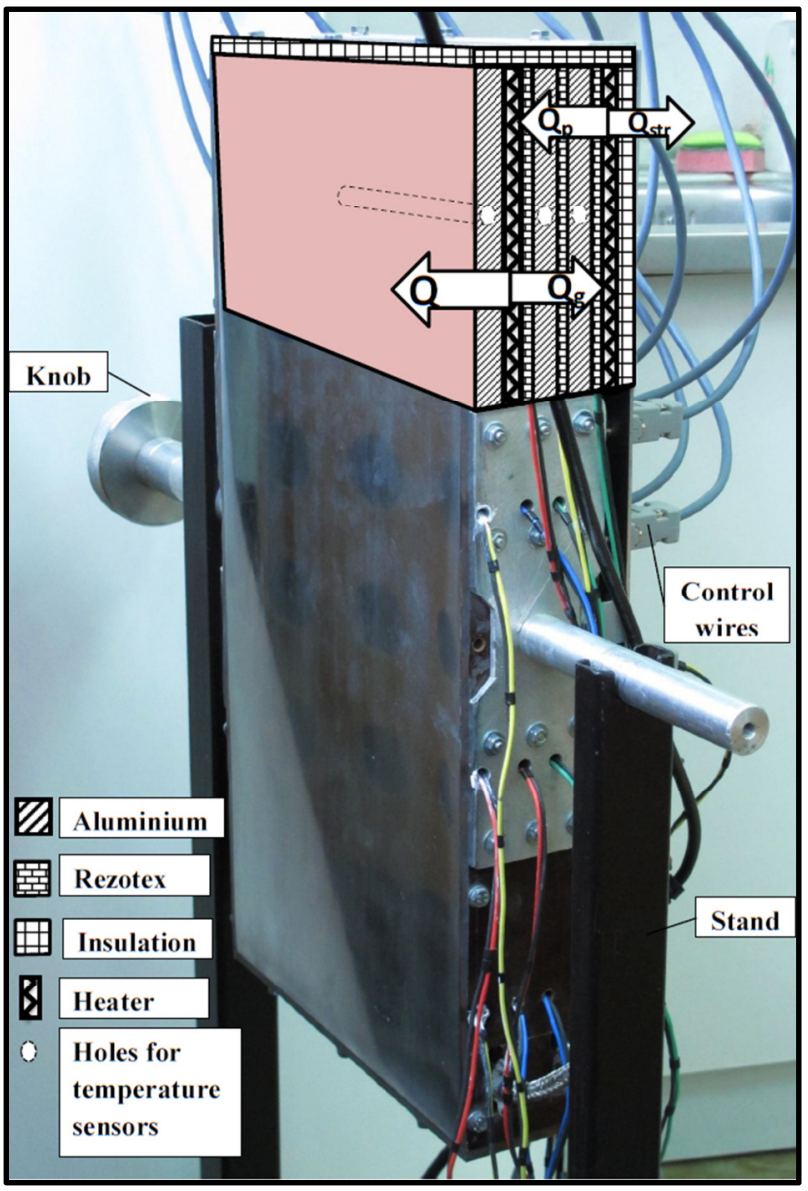

Figure 1. Heating plate with partial cross-section.

\subsection{Temperature fields visualization system}

For temperature field's documentation IR thermal camera Fluke Ti35 FlexCam® with temperature measurement accuracy given producer of $\leq 0.1^{\circ} \mathrm{C}$ has been used.

Infrared camera can record thermal radiation emitted by physical bodies in temperature range between -20 $100^{\circ} \mathrm{C}$ in mid-infrared bands (wavelengths from 1 to 15 $\mu \mathrm{m})$. These bands correspond with areas of high atmospheric permeability for infrared radiation. In case of atmospheric gases, absorption of radiation has selective character. Individual gases permeable radiation with characteristic wavelength ranges only and do not emit thermal radiation. Therefore, fabric mesh rinsed by hot air was used as a detector - in such case radiation could be recorded by IR camera.

IR camera was set at distance of $2 \mathrm{~m}$ from the plate, twice changing his orientation. Firstly, it was directed perpendicular to plane of heating surface to measure temperature and degree of uniformity (Fig. 3). Then it was set down parallel to heating surface and perpendicular to detector mesh (Fig. 2), to measure temperature fields on mesh (Fig. 4). The study was carried out in an isolated room in order to eliminate accidental air movement from ventilation and drafts.

Resulting IR photographs were analyzed using specially created computer program that calculates temperature gradients and corresponding values of Nusselt number.

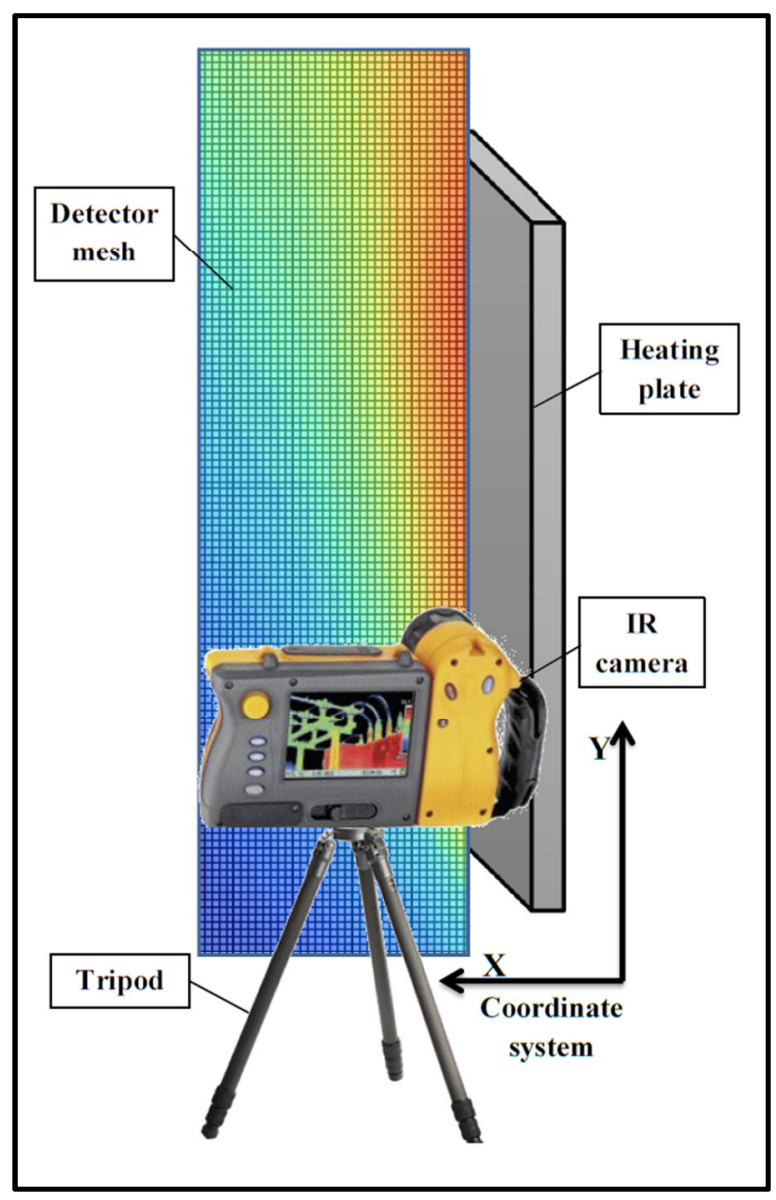

Figure 2. Scheme of heating plate with detector mesh and camera setup.

\section{Results and discussion}

For each testing case the desired temperature of the plate's surface has been set down and after reaching and determining thermal equilibrium the temperature and heating power have been measured and the photographs of mesh has been made. An example of temperature uniformity on the heated plate to $50^{\circ} \mathrm{C}$ is shown in Fig. 3 .

Figure 7 shows an example results obtained for plate temperature of: $30,40,50$ and $60^{\circ} \mathrm{C}$. It was not possible to show at the same time the temperature of heating surface and temperature fields. The IR camera focused its detection sensitivity on the plate, therefore temperature fields was invisible, or vice versa. On the image we can see the second case. Temperature fields of heat flux and temperature color of plate were numerically modeled from images obtained by IR camera perpendicular positioned to the plate (Fig. 2).

At initial temperature of the plate, at $30^{\circ} \mathrm{C}$ (Fig. 7a), none of characteristic structures can be seen. Temperature distribution seems to be linear with isotherms in vertical direction due to normal thermal air stratification in room. 


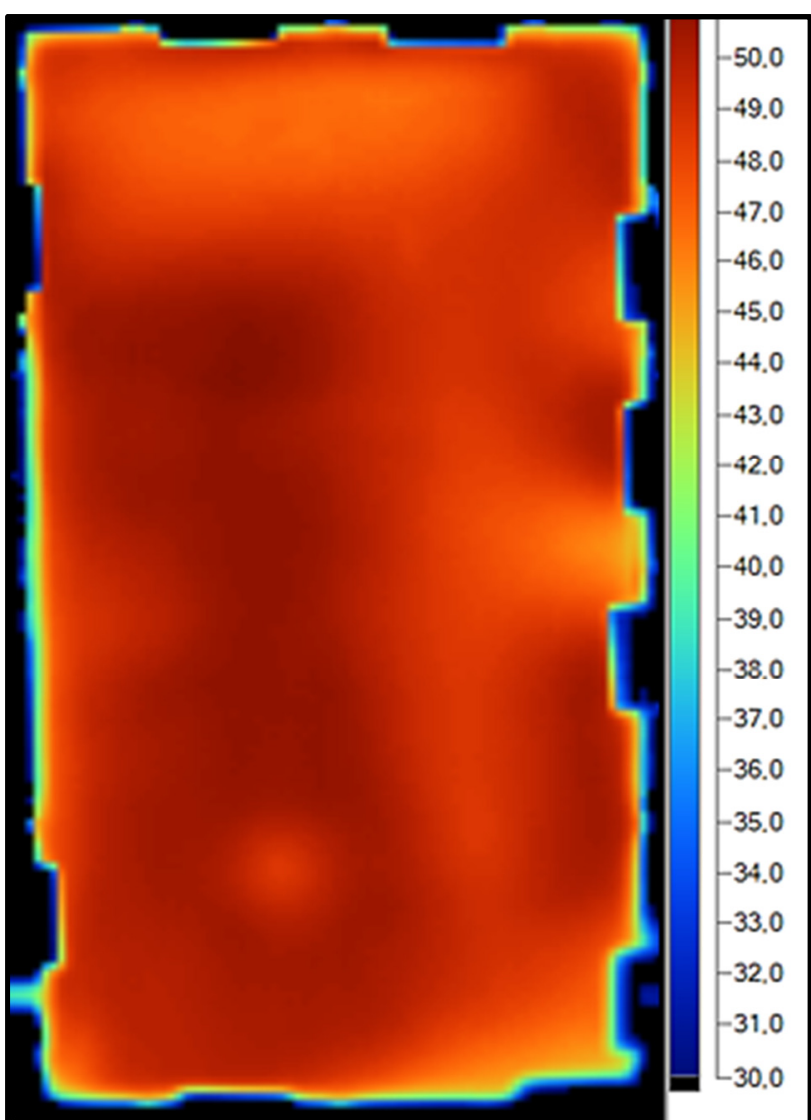

Figure 3. IR photograph of heating surface showing temperature uniformity at $50^{\circ} \mathrm{C}$.

When plate reaches a temperature of $40^{\circ} \mathrm{C}$ (Fig. 7b) one can observe explicit deviation of isotherms from horizontal direction, liquid begins to move in a vertical direction (upward), boundary layer is formed. Isotherms temperature values do not differ too much from the previous ones.

When heating plate temperature goes to a temperature of $50^{\circ} \mathrm{C}$ (Fig. $7 \mathrm{c}$ ) we observe a further sloping of isotherms - those which are closer to the plate are becoming practically vertical. Temperature gradient between extreme isotherms is larger.

Beginning from plate's temperature above a temperature of $60^{\circ} \mathrm{C}$ (Fig. 7d) increase density of isotherms in considered area can be observed with presence of hottest areas closest to the plate - isotherms takes a spherical shape.

Please note that temperature fields presented in Fig. 7 are both air and fiber mesh temperatures, because only mesh are able to emit radiation that can be observed using IR camera.

To avoid heat transfer by conduction from heating plate to mesh fibers, the mesh fiber should not be too thick or pressed onto heating surface. As a result, isotherms in Fig. 7 end at the edge of mesh. In fact they are extended further parallel to the heating surface as shown in enlarged detail of plate in Fig. 4 which illustrate hypothetical [8] course of dashed line. Isotherms in this area undergo concentration and distances between them

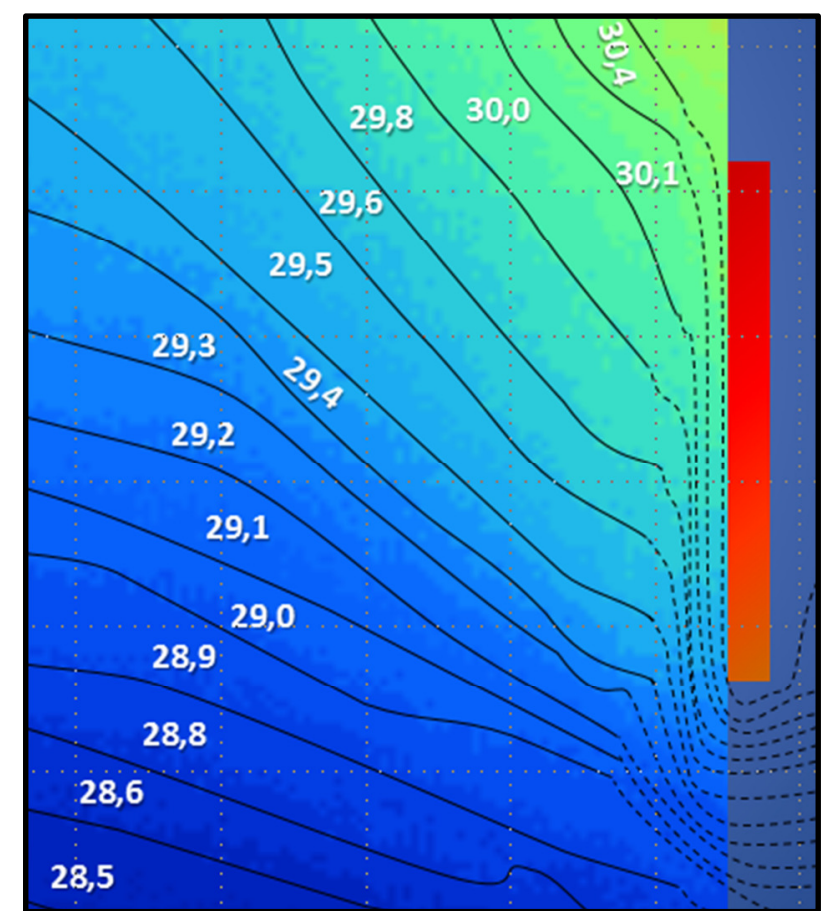

Figure 4. Hypotetical isoterms distrubution in vicinity of the plate.

are too small in relation to size of grid mesh that can be visualized using this method.

Examples of temperature profiles measured at half height of plate with surface temperature $\boldsymbol{T}_{\mathrm{w}}=30,40,50$ and $60^{\circ} \mathrm{C}$ are shown in Fig. 5. Tangent to each of these temperature profiles $\left(\partial \boldsymbol{T} /\left.\partial \boldsymbol{x}\right|_{x=0}\right)$ is experimentally determined value of local heat transfer coefficient $\boldsymbol{\alpha}_{\boldsymbol{y}}$ obtained by gradient method using IR camera. Results obtained in form of images and matrix of temperature and distance values was processed numerically.

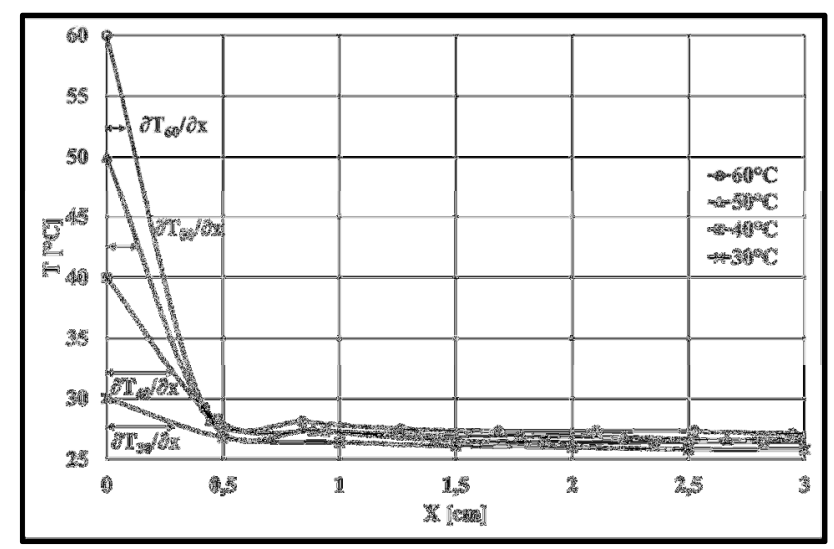

Figure 5. Temperature profiles at half height of plate.

The final result of these calculations conducted for specific preset temperature of surface $\boldsymbol{T}_{\boldsymbol{w}}$ is Nusselt number. Nusselt number is correlated with Rayleigh number determined for the course of experiment $(\Delta \boldsymbol{T})$ from equation (1). Results obtained from relation (7) with experimental points which from this relation was calculated and literature relations are shown in Fig. 6. 


$$
N u=0.647 R a^{0,236}\left(R^{2}=0.943\right)
$$

As shows analysis of Fig. 6 obtained results are consistent with results of other researchers and our other studies by us using balance method [9].

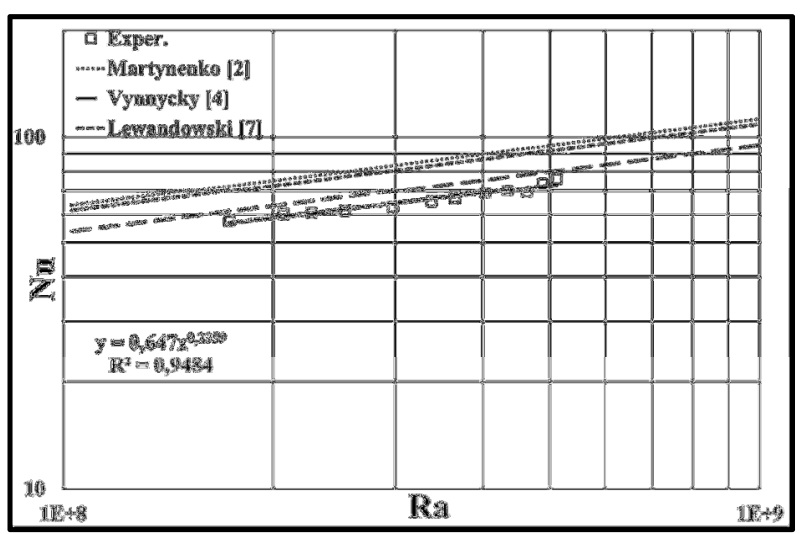

Figure 6. Comparison experimental points with literature data.

Table 1 compares values of $\boldsymbol{C}$ and $\boldsymbol{n}$ coefficients from equation (4) obtained by us with literature data.

Table 1. Comparison of $\mathrm{C}$ and $\mathrm{n}$ coefficients.

\begin{tabular}{|c|c|c|c|}
\hline Author & C & n & Ra range \\
\hline Experimental & 0,647 & 0,24 & $10^{8}<\mathrm{Ra}<10^{9}$ \\
\hline Martynenko [2] & 0,634 & 0,25 & $10^{2}<\mathrm{Ra}<10^{9}$ \\
\hline Vynnycky [4] & 0,535 & 0,25 & $\mathrm{Ra}=10^{6}$ \\
\hline $\begin{array}{c}\text { Lewandowski, } \\
\text { Kubski [7] }\end{array}$ & 0,613 & 0,25 & $10^{4}<\mathrm{Ra}<10^{8}$ \\
\hline $\begin{array}{c}\text { Denda, } \\
\text { Lewandowski } \\
\text { [9] }\end{array}$ & 0,491 & 0,25 & $10^{8}<\mathrm{Ra}<10^{9}$ \\
\hline
\end{tabular}

\section{Conclusions}

Our studies prove that with appropriate usage of IR camera, temperature distributions in boundary layer of heating surface can be achieved so precisely, that the field of local heat transfer coefficients can be determined. This allows calculating local and global Nusselt number values using gradient method. Literature data have positive verified correctness of new convection test method presented in this work using thermal imaging camera.

An advantage of this method is undoubtedly its simplicity which results in low cost of measurements implementation. Noteworthy is also its execution speed. After temperature equilibration, which usually takes a few minutes after performing thermal image and processing it through the program, we get data for entire investigated area immediately without time consuming sampling this area with traditional thermometers. This solution can be also applied to research large objects such as building walls, boilers surfaces and other power equipment.

\section{References}

1. S.W. Churchill, H.H. Chu, J. Heat Mass Transfer, 18, 1323-1329 (1975)

2. O.G. Martynenko, A.A. Berezovsky, Y.A. Sokovishin, Int. J.Heat Mass Transfer, 27, 869881 (1984)

3. J.D. Hellums, S.W. Churchill, AIChE Journal, 8, 690-692 (1962)

4. M. Vynnycky, S. Kimura, Int. J. Heat Mass Transfer, 39, 1067-1080 1(996)

5. J. Gile, J. Fluid Mech, 30, 371-384 (1967)

6. L. Zhao-Zhuang, W. Bu-Xuan, X. Jia-Yu Int. J. Heat Mass Transfer, 34, 2813-2818, (1991)

7. W. M. Lewandowski, P. Kubski, Wärme und Stoffübertragung, 18, 247-256 (1984)

8. K. Brodowicz, Int. J. Heat Mass Transfer, 2, 201-204, (1968)

9. W. M. Lewandowski, H. Denda, M. Ryms, E. Klugmann-Radziemska, Possibility of thermal imaging usage in studies of natural convection heat transfer from an isothermal vertical plate. Int. J.Heat Mass Transfer (in press) 


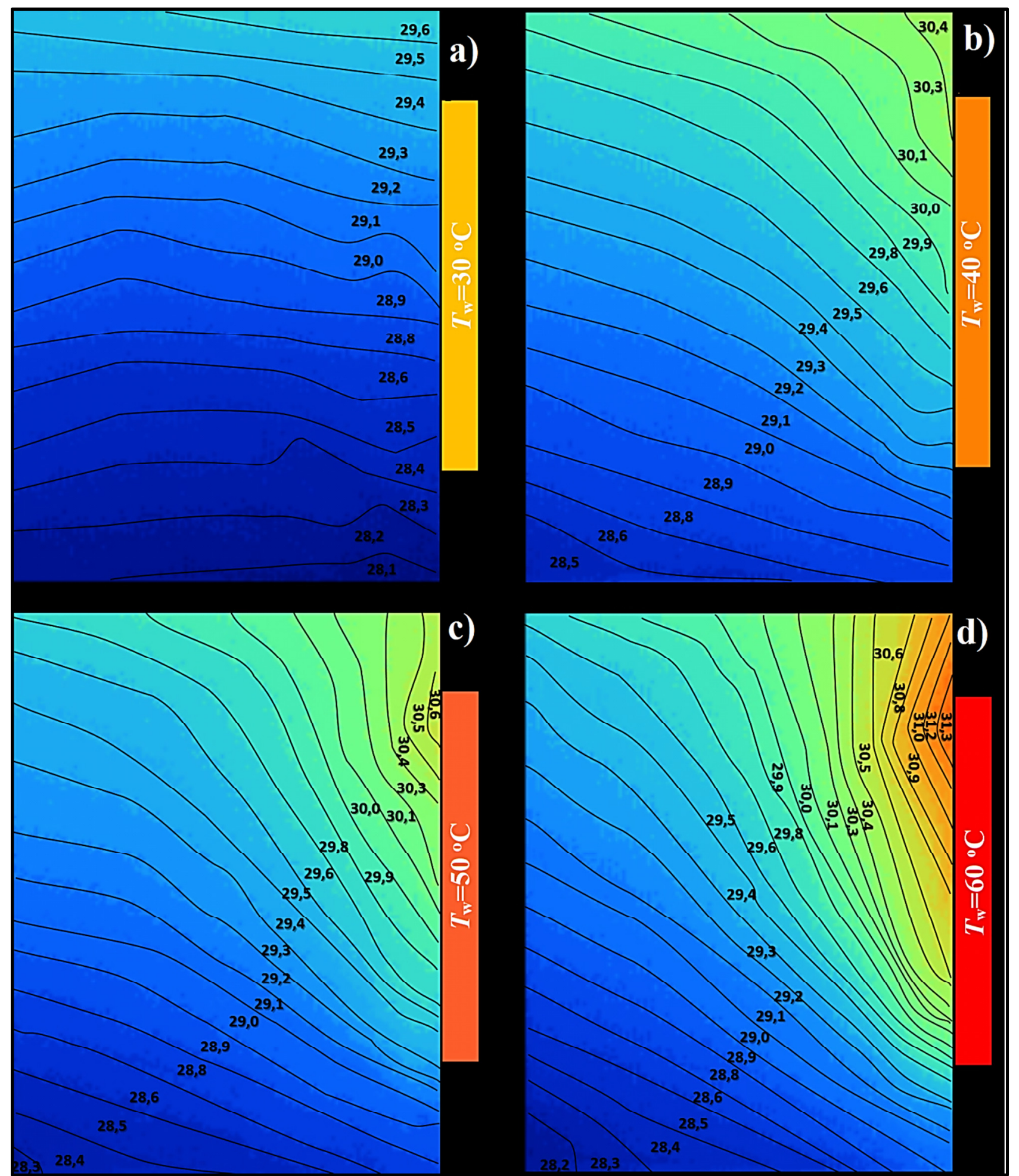

Figure 7. Cross sections of air layers with marked isotherms and temperature of plate. 